\title{
Revealing map reading strategies of physical geographic maps
}

\author{
Marketa Beitlova $^{\mathrm{a}, *, \text { Stanislav Popelka }}{ }^{\mathrm{a}}$ \\ a Palacký University Olomouc, Czech Republic, marketa.beitlova@gmail.com, stanislav.popelka@upol.cz \\ * Corresponding author
}

Keywords: Map reading strategy, Eye-tracking, Physical geographic map, School world atlas, Geographic education

\begin{abstract}
:
This contribution is focused on a qualitative exploration of map reading strategies based on eye-tracking data. The map reading strategy is defined as an iterative interaction between the user's mind and the map content. Map-reading strategies can be observed during completion of simple tasks designed based on the pyramid framework (Mennis et al., 2000), see Figure 1 .
\end{abstract}

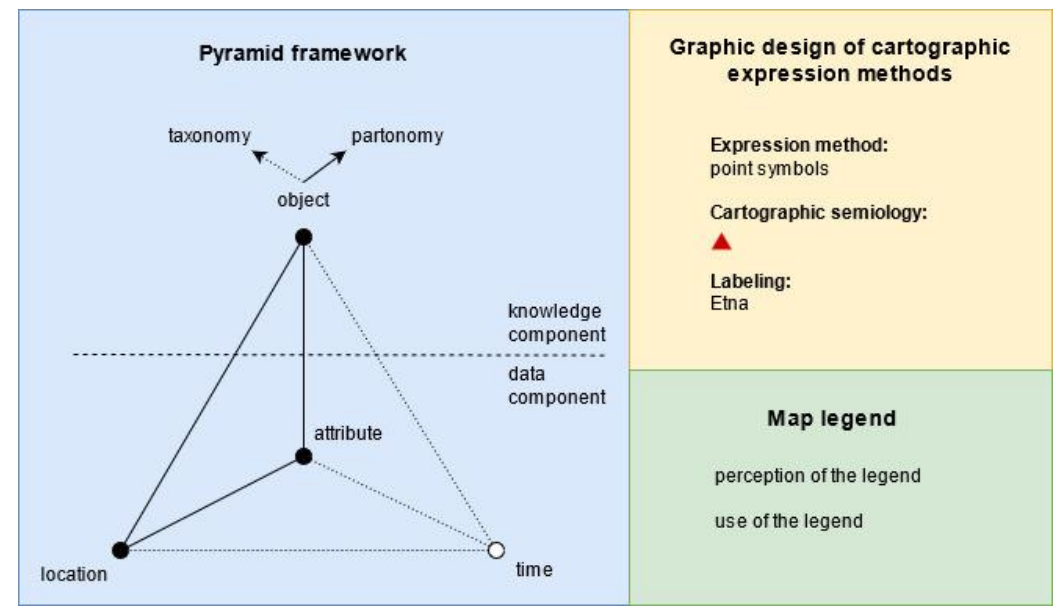

Figure 1. Key map elements for map reading.

A qualitative analysis of eye movements can be used to understand the process of extracting information from the map and identifying the dominant elements of the map that the respondent used during the task completion. By combining eye movements with the user behaviour, the way the map is read can be revealed. Identifying the dominant strategy allowed us to describe the cognitive abilities of map users and the level of their knowledge during the use of key map elements.

Twelve physical geographic maps from school world atlas were used as a stimuli. The respondents were 22 elementary school students and their geography teacher. The tasks for the eye-tracking experiment were constructed using a pyramid framework, graphical map variables, and map legend. The objects that the users should find in a map were selected based on an analysis of the school atlas index. Data were recorded using SMI RED 250 eye-tracker and analysed qualitatively.

The synthesis of the findings revealed the respondents' ability to use the key map elements and identified basic strategies for physical geographic maps reading. The findings were categorized based on knowledge of the location, perception of the legend, work with the legend, perception of the symbols, and perception of hypsometric tints. The most straightforward strategy was used by $21.7 \%$ of respondents who solved the tasks based on their knowledge. The second identified strategy was the use of the legend, which was observed for $31.5 \%$ of the respondents. However, only $13 \%$ of respondents were able to find the correct answer using the legend. The third strategy was the perception of hypsometric tints. For these tasks, it was found that respondents spent $34.8 \%$ of the time looking at the correct interval of hypsometric or bathymetric tints. In tasks where the perception of hypsometric and bathymetric tints could be applied, respondents spent $50.4 \%$ of the observation time in the correct areas in the map (e.g., lowlands, highlands). The fourth revealed strategy is the perception of the symbols. Based on a synthesis of key elements and user behaviour, combinations of map reading ways were also identified.

The study revealed students' abilities to use their knowledge of each element in map reading. Experiment analysis identified the dominant strategies used by students and their similarity with the teacher's strategy. Finally, it verified the appropriateness of using the pyramid framework in map reading strategy detection. 